\title{
ESTUDIO DE LOS HONGOS QUE FRUCTIFICAN EN LA VEGETACION RELICTA DE ABIES PINSAPO BOISS., EN ESPAÑA PENINSULAR. II. ASCOMYCOTINA. II
}

\author{
Juana María LARIOS, Mario HONRUBIA \& Gabriel MORENO
}

\begin{abstract}
RESUMEN: Se citan 41 taxones de la subdivisión Ascomycotina procedentes de la vegetación relicta de Abies pinsapo Boiss. en las provincias de Málaga y Cádiz. Es de resaltar la presencia de Aphiorhynchostoma curreyi (Rabenh.) Müller, Cucurbitaria berberidis (Pers.: Fries) Gray, Sydowia polispora (Bref. \& v. Tavel) Müller, Glonium graphicum (Fries) Duby, Caloscypha fulgens (Pers.) Boudier, Coronophora abietina Fuck. y Rosellinia tassiana De Not. Se incluyen observaciones de tipo morfológico y taxonómico y se ilustran con figuras originales la mayoria de los táxones tratados.
\end{abstract}

SUMMARY: 41 taxa of Ascomycotina collected in the relict vegetation of Abies pinsapo Boiss., in the provinces of Málaga and Cádiz, are showed here. One should note the presence of: Apiorhynchostoma curreyi (Rabenh.) Müller, Cucurbitaria berberidis (Pers.: Fries) Gray, Sydowia polispora (Bref. E v. Tavel) Müller, Glonium graphicum (Fries) Duby, Caloscypha fulgens (pers.) Boudier, Coronophora abietina Fuck. and Rosellinia tassiana De Not. Iaxonomical and morphological observations are given. Original plates are included in almost all the studied taxa.

Palabras clave: Ascomycotina, Abies pinsapo. España.

Key words: Ascomycotina, Abies pinsapo. Spain.

\section{INTRODUCCION}

Continuando con el estudio de los Ascomycotina que fructifican en la vegetación relicta de Abies pinsapo Boiss, iniciado por Larios et a1. (1986), se citan 41 taxones recolectados en los pinsapares de Cádiz y Málaga.

Se ha seguido la ordenación taxonómica propuesta por Eriksson \& Hawksworth (1985). Se comenta la posición incierta de algunas de las especies tratadas.

Para cada cita se indica: localidad de procedencia, recolector, fecha de recolección, número de registro y sustrato donde se ha encontrado. El material estudiado se halla depositado en el Herbario de la Universidad de Murcia (MUB). 


\section{CATALOGO DE ESPECIES}

\section{AMPHYSPHAERIALES}

Amphisphaeriaceae Winter

Apiorhynchostoma curreyi (Rabenh.) Müller (Fig. 1:A-D)

Material estudiado: Sierra del Pinar, Grazalema (Cádiz). Leg. JL. 2-V-86. JL. 726்1/22-XI-86. JL. 7270.

Hábitat: En madera muerta, descortezada, de Abies pinsapo.

Observaciones: El género Apiorhynchostoma Petrak se caracteriza por la presencia de peritecios inmersos en la corteza, con hifas de color gris que rodean a los ostíolos emergentes, a semejanza de un clípeo (Wehmeyer, 1975; Dennis, 1978). Las ascósporas de A. curreyi son de color pardo, elipsoideo-cilindricas, de 18-25 x 6-8 $\mu \mathrm{m}$; con dos septos: uno. mediano superior y el segundo que delimita una célula hialina basal. La célula superior posee un pequeño apéndice hialino. A. curreyi es característica de madera de coniferas (Dennis, 1978; Ellis \& Ellis, 1985).

\section{DIATRYPALES}

Diatrypaceae Nits.

Diatrype stigma (Hoffmann: Fries) Fries

Material estudiado: Sierra del Pinar, Grazalema (Cádiz). Leg. JL. 11-XI-83. JL. 7206/2-V-86. JL. 7213. JL. 7264/ Pinsapar del Cortijo de la Nava de San Luis, Ronda (Málaga). Leg. JL. 1-V-86. JL. 7291.

Hábitat: Sobre ramas muertas corticadas, de Quercus faginea y Quercus rotundifolia.

Observaciones: Especie muy común sobre ramas de Quercus sp. pl., ampliamente distribuida en la Península Ibérica. Citada por Beltault (1974) de Grazalema, sobre quejigo.

Diatrypella quercina (Pers.: Fries) Cooke

Material estudiado: Sierra del Pinar, Grazalema (Cádiz). Leg. JL. 14-XI-83. JL. 7247/2-V-86. JL. 7210.

Hábitat: En ramas muertas corticadas de Quercus faginea.

Observaciones: al igual que el anterior taxon, D. quercina es de amplia distribución en España. Citada por Bertault (1974) sobre Q. faginea en las cercanías de Grazalema.

Eutypa acharii Tulasne

Material estudiado: Los Reales, Sierra Bermeja, Estepona (Málaga). Leg. JL. 1-V-86. JL. 7239.

Hábitat: En ramas muertas de pinsapo.

Observaciones: Estromas efusos, erumpentes, con aspecto de largas pústulas sobre el sustrato. Peritecios inmersos en el estroma, con ostíolos emergentes, engrosados, sulcados.

Según Breitenbach \& Franzklin (1981) "una rama con este ascomiceto tiene aspecto de estar quemada, con puntitos negros que son los ostíolos".

Berlese (1968) cita este taxon sobre una amplia variedad de sustratos.

\section{DOTHIDEALES}

Cucurbitariaceae Winter

Cucurbitaria berberidis (Pers.: Fries) Gray (Fig. 1: E-F)

Material estudiado: Sierra de Las Nieves, Ronda (Málaga). Leg. JL. 1-V-86. JL. 7297.

Hábitat: En ramas muertas de Abies pinsapo. 

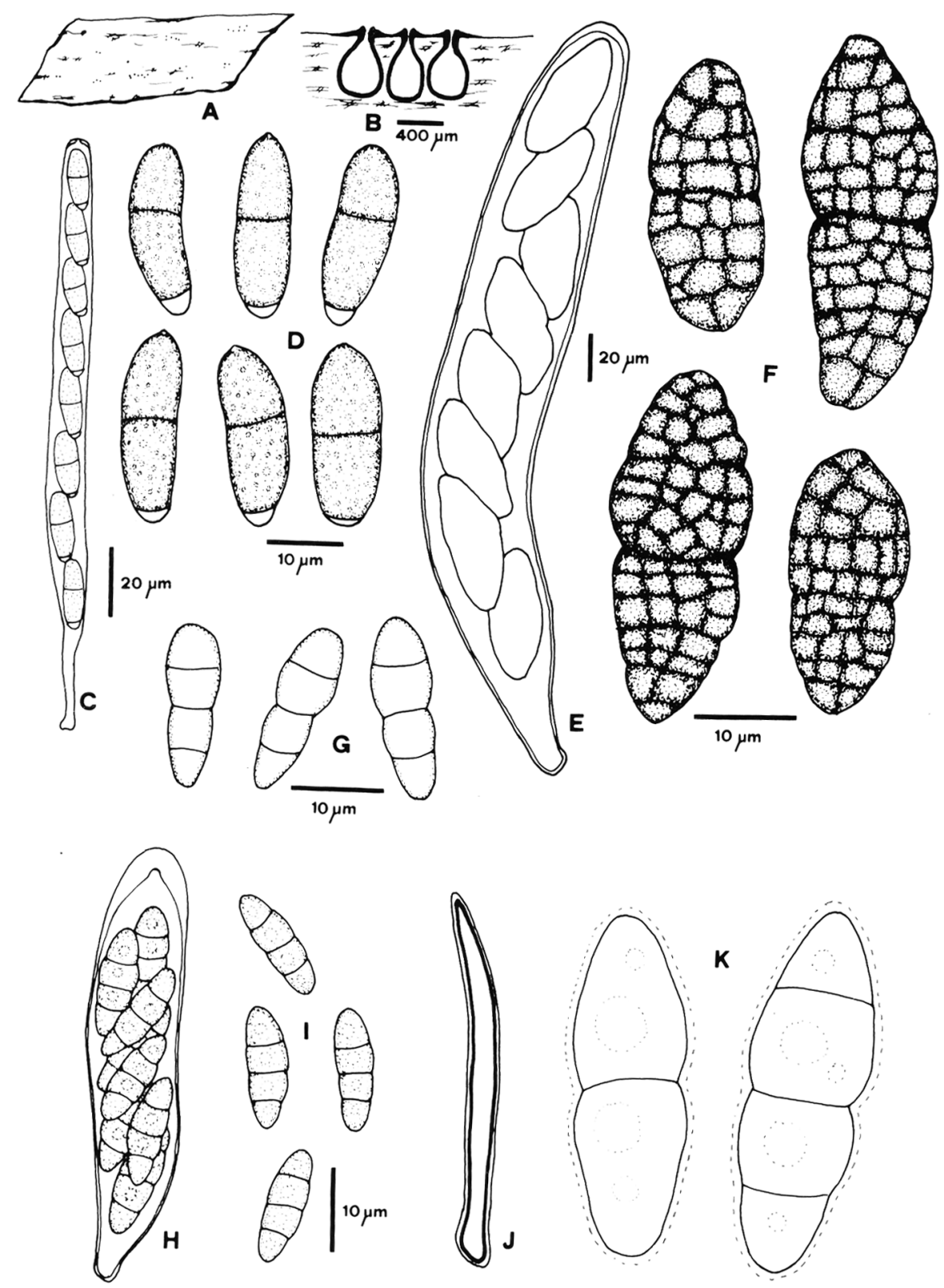

FIGURA 1. A-D: Apiorhynchostoma curreyi. A: Aspecto general de los ascomas sobre el sustrato. 8: Corte transversal de los ascomas. C: Asco. D: Ascósporas. E-F: Cucurbitaria berberidis. E: Asco. F: Ascósporas. G: Sydowia polispora: Ascósporas. H-J: Herpotrichiella pilosella. H: Asco. I: Ascósporas. J: Pelo de la pared del ascoma. K: Massarina cisti: Ascósporas. 
Observaciones: Ascomas en grupos que emergen rompiendo la corteza, con pared gruesa y rugosa.

El material estudiado coincide con la descripción dada por Mirza (1968) de C. berberidis, excepto en el número de septos de las ascósporas que es, en el material de la Sierra de las Nieves de hasta 14 septos transversales y 3-5 longitudinales, incompletos. Mirza indica 4-9-(12) septos transversales y 3 longitudinales.

Dothioraceae Theis. \& Sydow

Sydowia polyspora (Bref. \& Tavel) Muiller (Fig. 1:G)

Material estudiado: Cortijo de la Nava de San Luis. Ronda (Málaga). Leg. JL. 1-V-86. JL. 7251.

Hábitat: En ramas muertas de pinsapo.

Observaciones: Sydowia Bres. se caracteriza por poseer ascos polisporados con ascósporas hialinas pluriseptadas. Las ascósporas de S. polyspora son triseptadas, claviformes. Munk (1957) indica ascos 16-esporados, mientras que Barr (1972) y Sivanesan (1984) 24-32-esporados. En el material estudiado se observan ascos 16-24-esporados.

Saprófito o parásito, sobre hojas y ramas de coníferas (Barr, 1972; Sivanesan, 1984). Los ascomas, aislados o en grupos, se sitúan en las cicatrices foliares, rompen la peridermis al emerger y forman una especie de chancro. Referente a esto, Smerlis (1970a, in Barr, 1972) señala el carácter patógeno de este hongo en cierto número de Gimnospermas.

Herpotrichiellaceae Munk

Dictyotrichiella sp. (Fig. 2:A-C)

Material estudiado: Pinsapar de la Sierra del Pinar, Grazalema (Cádiz). Leg. MH. 14-XI-83. JL. 7067. / Pinsapar del Cortijo de la Nava de San Luis, Ronda (Málaga). Leg. JL. 22-XI-84. JL. 7077 / Pinsapar de la Sierra de las Nieves, Ronda (Málaga). Leg. JL. 23-XI-86. JL. 729. JL. 7282. JL. 7283.

Hábitat: Sobre ramas muertas, descortezadas de Abies pinsapo.

Observaciones: Ascomas de 100-200 $\mu \mathrm{m}$ de diámetro, de dispersos a gregarios, semiinmersos cuando jóvenes, superficiales cuando maduros, subglobosos, de color negro, sujetos al sustrato por hifas de color pardo oscuro, cubiertos con pelos dispersos, agudos, de 30-55 x 3-3,5 $\mu \mathrm{m}$, de color pardo oscuro.

Pared del ascoma de 20-30 $\mu \mathrm{m}$ de grosor, formado por tejido pseudoparenquimático de textura angular, con células de 3-7 $\mu \mathrm{m}$ de diámetro, de pared gruesa.

Ascos de 60-75 x 14-20 $\mu \mathrm{m}$, obclaviformes, pedicelados, bitunicados, octosporados.

Ascósporas de 12-20 x 5-7 $\mu \mathrm{m}$, dispuestas desordenadamente en el asco, elipsoideo-fusiformes, con la mitad inferior más estrecha, con 5-7 septos transversales y un septo longitudinal incompleto, de color pardo oliváceo.

Dictyotrichiella Munk es un género de la familia Herpotrichiellaceae Munk que presenta ascos octosporados y ascósporas muriformes. Según Arx \& Muiller (1975) incluye tres especies: D. pulcherrima Munk, D. mansonii Schol-Schwarz y D. potentillae Müller. Ninguna de ellas coincide con el material estudiado.

Según Munk (in litt.), el material estudiado debe incluirse en este género y posiblemente representa un taxon nuevo para la ciencia.

Herpotrichiella pilosella (Karsten) Munk (Fig. 1:H-J.)

Material estudiado: Sierra del Pinar, Grazalema (Cádiz). Leg. JL. 22-XI-86. JL. 7275. Sierra de las Nieves, Ronda (Málaga). Leg. JL. 23-XI-86. JL. 7281 . 


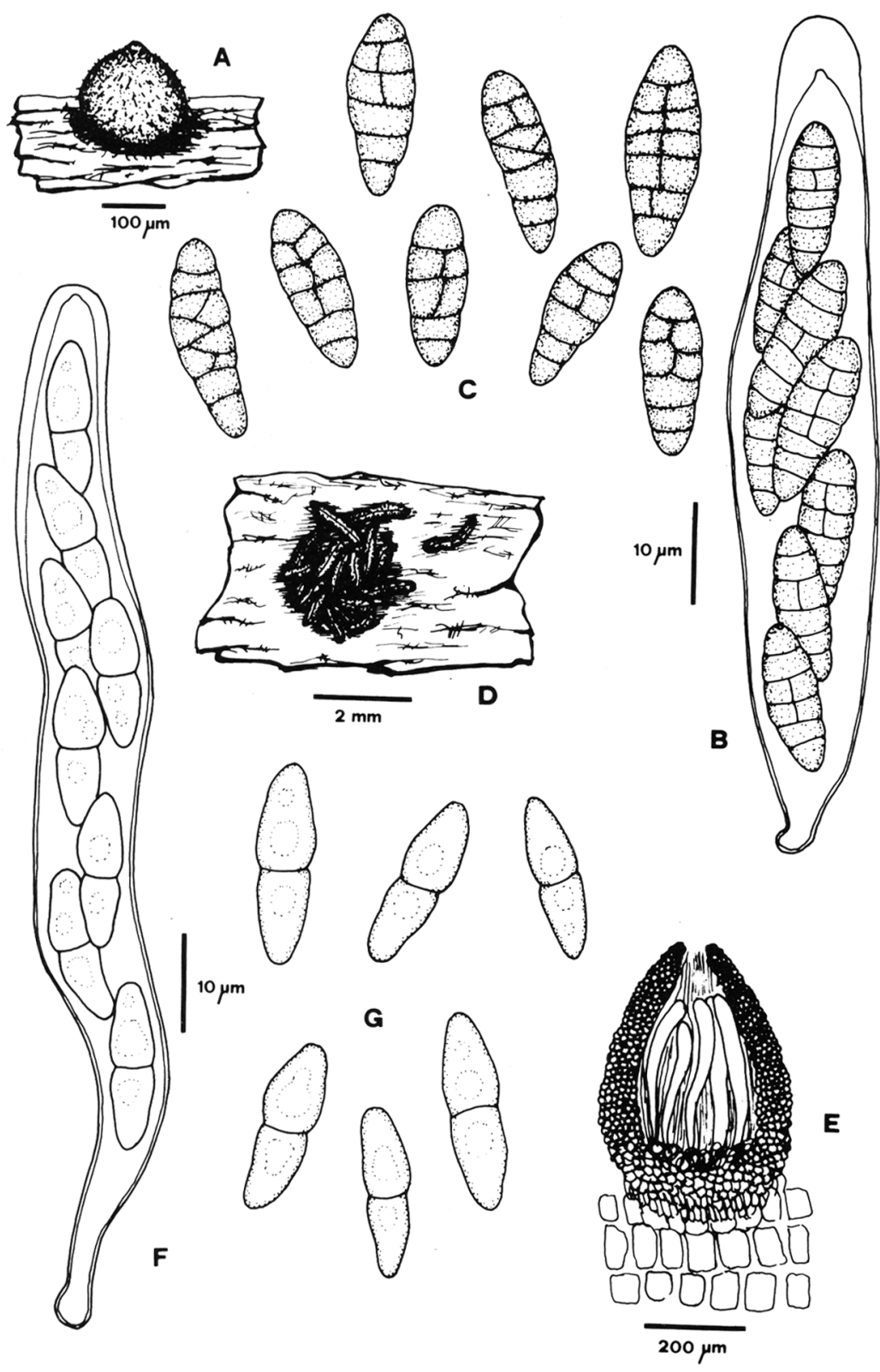

FIgURA 2. A-C: Dictyotrichiella sp. A: Ascoma. B: Asco. C: Ascósporas. D-G: Glonium graphicum. D: Histerotecios sobre el sustrato. E: Corte de un histerotecio. F: Asco. G: Ascósporas. 
Hábitat: Sobre madera muerta, en avanzado estado de descomposición, de Quercus faginea.

Observaciones: Ascomas de 100-125 $\mu \mathrm{m}$ de diámetro, cubiertos de pelos

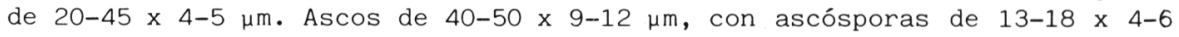
$\mu \mathrm{m}$, de color pardo-oliváceo, triseptadas.

Este taxon es común sobre madera muerta de árboles caducifolios (Holm, 1977).

Hysteriaceae Chev.

Glonium graphicum (Fries) Duby (Fig. 2:D-G)

Material estudiado: Los Reales, Sierra Bermeja, Estepona (Málaga). Leg. JL. 1-V-86. JL. 7244.

Hábitat: Sobre corteza de Pinus sp.

Observaciones: Histerotecios de 0,75-1,5 x 0,25-04 $\mu \mathrm{m}$ sinuosos, bifurcados o ramificados, aislados o densamente agrupados sobre un bien desarrollado subículo, cilíndricos, de color negro, con ranura algo prominente, a modo de quilla, a lo largo del ascoma. Pared del histerotecio gruesa, formada por pseudoparénquima de textura angular; sus células presentan pared gruesa, excepto hacia la base, donde la tienen más delgada. Subículo formado por hifas de color pardo, tabicadas.

Ascos de 80-110 x 10-13 $\mu \mathrm{m}$, cilíndricos, pedicelados, octosporados, bitunicados.

Ascósporas de 15-20 x 5-6 $\mu \mathrm{m}$, biseriadas, elpsoideo-fusiformes, hialinas uniseptadas, célula superior más ancha que la inferior, ambas gutuladas.

Este taxon, poco frecuente, se encuentra sobre madera y corteza de Pinus y Juniperus (Zogg, 1962).

Hysterium angustatum Alb. \& Schwein.

Material estudiado: Sierra del Pinar, Grazalema (Cádiz). Leg. JL . 14-XI-83. JL. 7200 .

Hábitat: Sobre rama muerta, descortezada de Quercus faginea.

Observaciones: El material estudiado concuerda con la descripción dada por Zogg (1962) para H. angustatum. Esta especie ha sido citada de distintos puntos de España.

Lophiostomataceae Nits.

Lophiostoma sp. (Fig. 3:A)

Material estudiado: Sierra de las Nieves, Yunquera (Málaga). Leg. JL. 23-XI-84. JL. 7073.

Hábitat: Sobre madera muerta no identificada.

Observaciones: Ascomas de 375-600 $\mu \mathrm{m}$ de diámetro, gregarios, erumpentes, subglobosos, de color negro, con la pared esclerotizada. Papila ostiolar comprimida lateralmente y abierta por una ranura longitudinal, en ocasiones de la misma anchura que el resto del ascoma.

Ascos de 100-130 x 12-15 $\mathrm{m}$, cilíndricos, estipitados, bitunicados, octosporados.

Ascósporas de 35-43 x 6-7 $\mu \mathrm{m}$, biseriadas, fusiformes, hialinas, ligeramente curvadas, de pared gruesa, con 9-13 septos transversales y un septo longitudinal en las 2-3 células centrales, gutuladas. Cuando jóvenes, presentan vaina.

Berlese (1894) propuso el género Lophidiopsis, en el que incluía lofiostomatáceos con ascósporas muriformes, hialinas. Arx \& Muiller (1975) y Hawksworth et al. (1983), consideran Lophidiopsis Berl. sinónimo de Lophiostoma Ces. \& De Not.

El material estudiado no concuerda con ninguna de las especies conocidas de Lophiostoma. Las ascósporas son próximas a las de L. alpigenum Fuck., pero poseen un septo longitudinal en las células centrales, lo que 

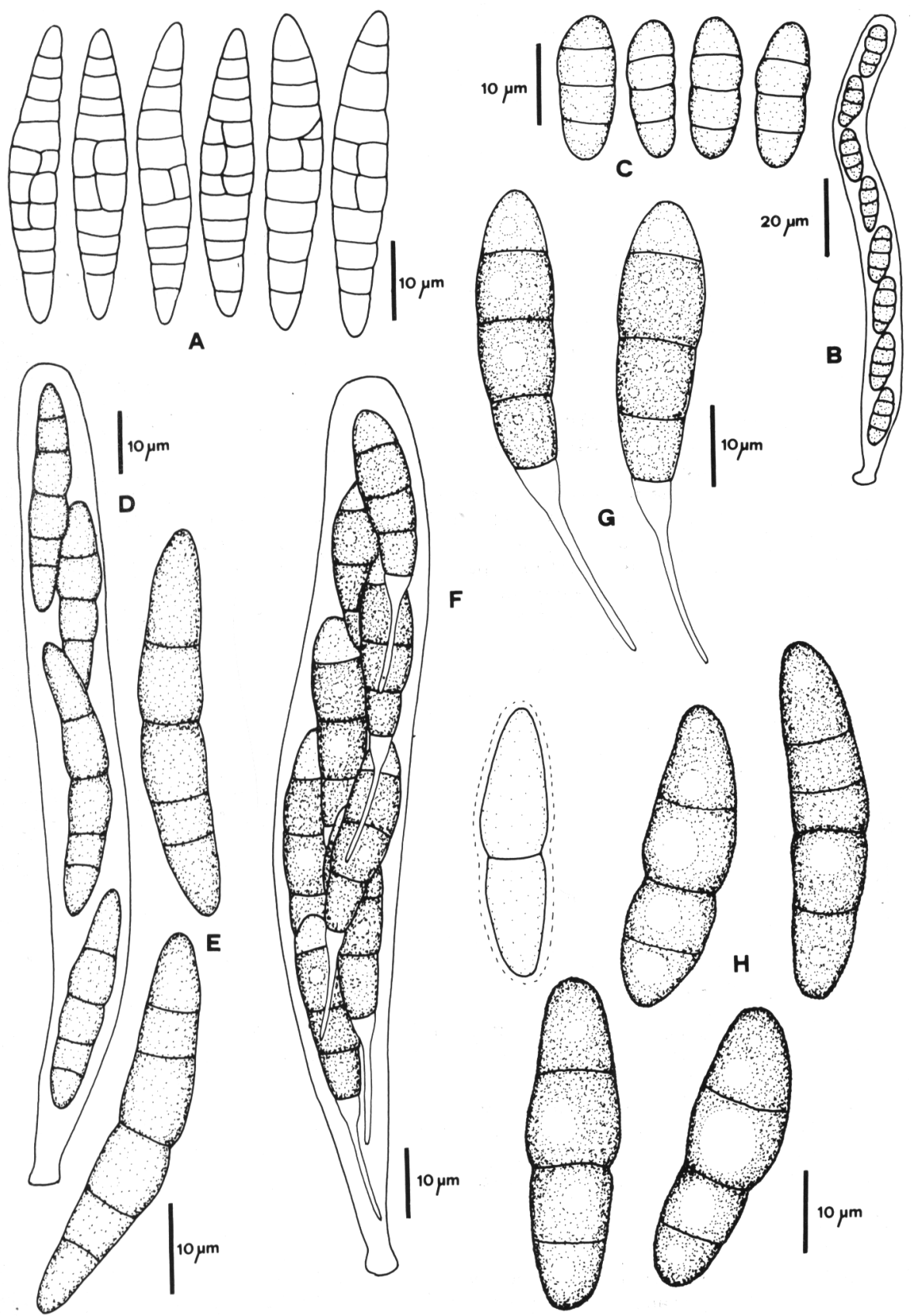

FIgURA 3. A: Lophiostoma sp.: Ascósporas. B-C: Lophiostoma quadrinucleatum var. triseptatum. B: Asco. C: Ascósporas. D-E: Lophiostoma subcorticale. D: Asco. E: Ascósporas. F-G: Rebenstichia unicaudata. F: Asco. G: Ascósporas. H: Aplanchnonema pupula: Ascósporas. 
no ocurre en la especie de Fuckel. Según Sivanesan (in litt.), posiblemente representa un taxon nuevo.

Lophiostoma quadrinucleatum var. triseptatum (Peck) Chesters \& Bell (Fig. 3:B-C)

Material estudiado: Sierra de las Nieves, Yunquera (Málaga). Leg. A. Aparicio. 23-XI-86. JL. 7287.

Hábitat: Raíz muerta de Ulex baeticus.

Observaciones: Chesters \& Bell (1970) reúnen, con el nombre de L. quadrinucleatum var. triseptatum, a L. pruni Ell. \& Ev., L. triseptatum Peck y L. triseptatum var. pleuriseptatum Ell. \& Ev. Según estos autores, la diferencia entre tales táxones no es suficiente para considerarlos con rango de especie. Piensan que se trata de una especie variable que da lugar, por un lado la var. triseptatum y por otro la var. quadrinucleatum, siendo L. pruni y L. triseptatum formas intermedias.

La var. triseptatum difiere del tipo por el menor tamaño de sus ascósporas.

Lophiostoma subcorticale Fuck. (Fig. 3:D-E)

Material estudiado: Sierra del Pinar, Grazalema (Cádiz). Leg. JL. 22-XI-86. JL. 7272 .

Hábitat: En ramas muertas de Quercus faginea.

Observaciones: Chesters \& Bell (1970) indican que este taxon posee ascósporas al principio hialinas, con 1 septo transversal que cuando alcanzan la madurez se vuelven pardas y 5-septadas. En el material estudiado, llegan a ser 3-5-septadas en el asco y de color pardo claro, más oscuro una vez que son expulsadas. De acuerdo con estos autores, la mayoría de los ascos contienen menos de 8 ascósporas y usualmente son tetrasporados.

\section{Massarinaceae Munk}

Massarina cisti Bose (Fig. $1: \mathrm{K}$ )

Material estudiado: Pinsapar del Cortijo de la Nava de San Luis, Ronda (Málaga). Leg. JL. 22-XI-84. JL. 7256.

Hábitat: En ramas muertas, descortezadas, de Cistus sp.

Observaciones: Taxon caracterizado por sus ascósporas de 30-36 x 8,5-12 $\mu \mathrm{m}$, uniseptadas al principio, finalmente triseptadas, constrictas, hialinas y con una vaina gelatinosa.

Se desarrolla sobre Cistus sp. (Sivanesan, 1984).

\section{Pleomassariaceae Barr}

Splanchnonema pupula (Fries: Fries) Kuntze (Fig. 3:H)

Material estudiado: Sierra de las Nieves, Ronda (Málaga). Leg. JL. 13-XI-83. JL. 7061. JL. 7062.

Hábitat: En rama de pinsapo muerta, descortezada.

Observaciones: Los ejemplares coinciden con la descripción de Barr (1982) y Sivanesan (1984) para Splanchnonema pupula. Munk (1957) denomina a esta especie Stigmatomassaria pupula (Fries) Munk.

Tubeufiaceae Barr

Rebenstichia unicaudata (Berk. \& Br.) Sacc. (Fig. 3:F-G)

Material estudiado: Sierra de Zafalgar, Grazalema (Cádiz). Leg. JL. 2-V-86. JL. 7222.

Hábitat: Sobre corteza muerta, desconocida.

Observaciones: Taxon caracterizado por sus ascósporas de 20-29 x 7-8 $\mu \mathrm{m}$, con $3(-4)$ septos transversales, de color pardo verdoso, gutuladas, célula superior subhialina, apéndice basal hialino de $6 \times 3-4 \mu \mathrm{m}$ en la 


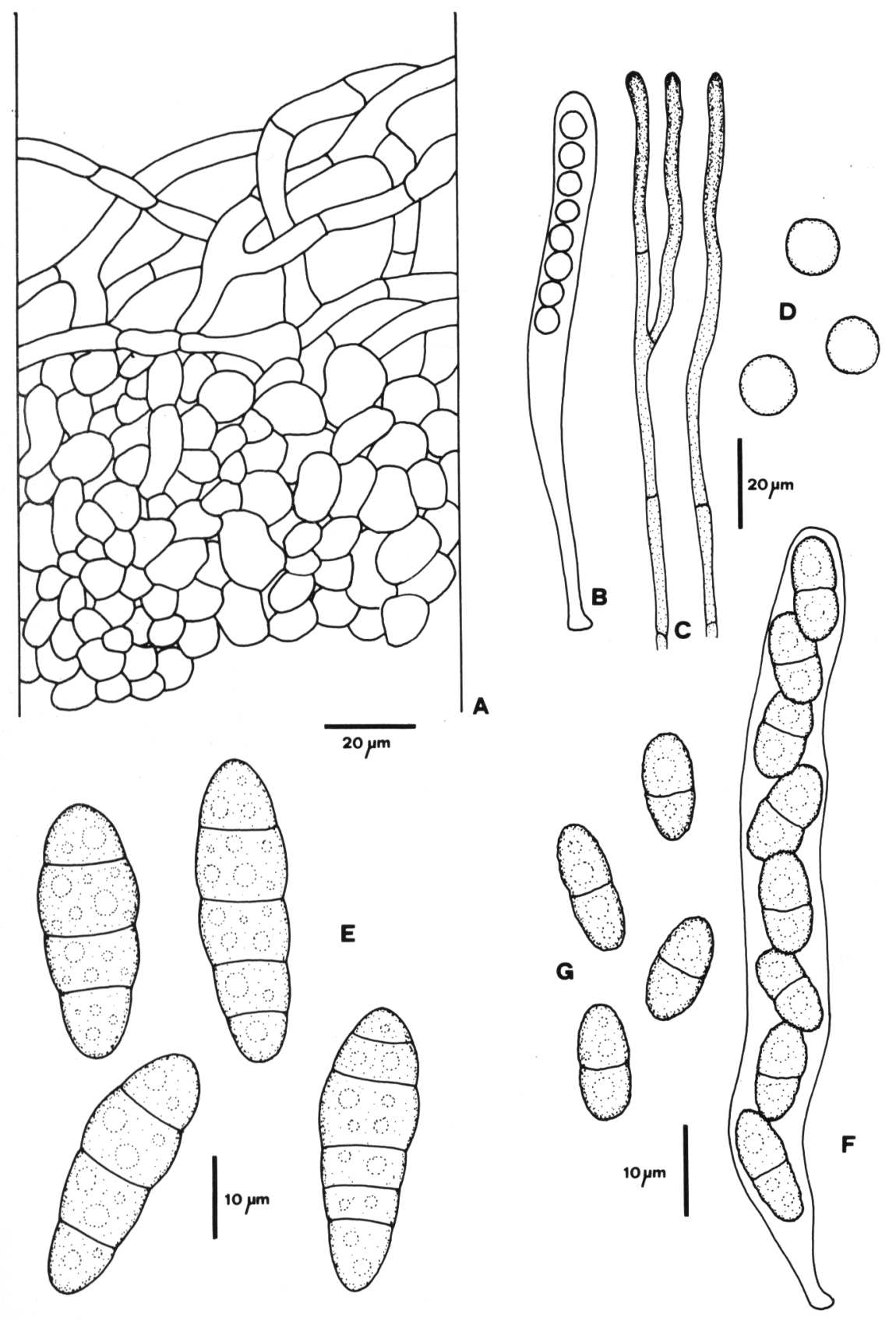

FIGURA 4. A-D: Caloscypha fulgens. A: Sección transversal del apotecio. B: Asco. C: Paráfisis. D: Ascósporas. E: Odontotrema inclusum: Ascósporas. F-G: Nectria flavoviridis. F: Asco. G: Ascósporas. 
parte engrosada y 10-16 x 1-1,5 $\mu \mathrm{m}$ en la parte inferior, filiforme.

De acuerdo con Saccardo (1883), R. unicaudata es la única especie de este género con la célula superior subhialina. También indica un tamaño de ascósporas ligeramente inferior $(18-21 \times 6 \mu \mathrm{m})$ al observado en nuestro material.

\section{HIPOCREALES}

Hypocreaceae De Not.

Nectria flavoviridis (Fuckel) Wollenweber (Fig. 4:F-G)

Material estudiado: Sierra de Zafalgar, Grazalema (Cádiz). Leg. JL. 2-V-86. JL. 7269.

Hábitat: Sobre viejos estromas de Diatrype sp. en ramas muertas, corticadas de Quercus faginea.

Observaciones: N. flavoviridis es un taxon próximo a N. episphaeria de la que difiere por el mayor tamaño de peritecios, ascos y ascósporas (Booth, 1959) citada por Bertault (1974) del pinar de San Cristobal (Cádiz).

\section{OSTROPALES}

Odontotremataceae Hawkw. \& Sherw.

Odontotrema inclusum (Karst.) (Fig. 4:E)

Material estudiado: Sierra de las Nieves, Yunquera (Málaga). Leg. JL. 23-XI-84. JL. 7072 / 23-XI-86. JL. 7286 / Sierra del Pinar, Grazalema (Cádiz). Leg. JL.'22-XI-86. JL. 7274.

Hábitat: En madera muerta, descortezada de Abies pinsapo.

Observaciones: El encuadre taxonómico de esta especie es discutido por Holm \& Holm (1977), que lo incluyen en el orden Ostropales.

Stictidaceae Fries

Stictis radiata Pers ex S.F. Gray

Material estudiado: Pinsapar de la Sierra de las Nieves, Yunquera (Málaga). Leg. JL. 3-V-86. JL. 7241.

Hábitat: En ramas muertas, descortezadas de pinsapo.

Observaciones: Taxon macroscópicamente semejante a S. stellata Wall., que crece sobre sustrato herbáceo. Esta última posee ascósporas más estrechas que $\mathrm{S}$. radiata $(1,5-2 \mu \mathrm{m}$ y $2-2,5 \mu \mathrm{m}$ respectivamente) (Dennis, 1978 ).

\section{PEZIZALES}

Helvellaceae Fries

Discina melaleuca Bres.

Material estudiado: Pinsapar del Cortijo de la Nava de San Luis, Ronda (Málaga). Leg. JL. 1-V-86. JL. 7298.

Hábitat: Sobre tierra, al borde de un camino.

Observaciones: Especie frecuente, típica de bosques planifolios y coníferas, al borde de caminos o en claros (Breitenbach \& Franzlin, 1981).

Helvella lacunosa Afz.: Fries

Material estudiado: Pinsapar de la Sierra de las Nieves, Yunquera (Málaga). Leg. JL. 23-XI-86. JL. 7278.

Hábitat: Sobre tierra.

Observaciones: Común, encontrada abundantemente en la zona de estudio. 

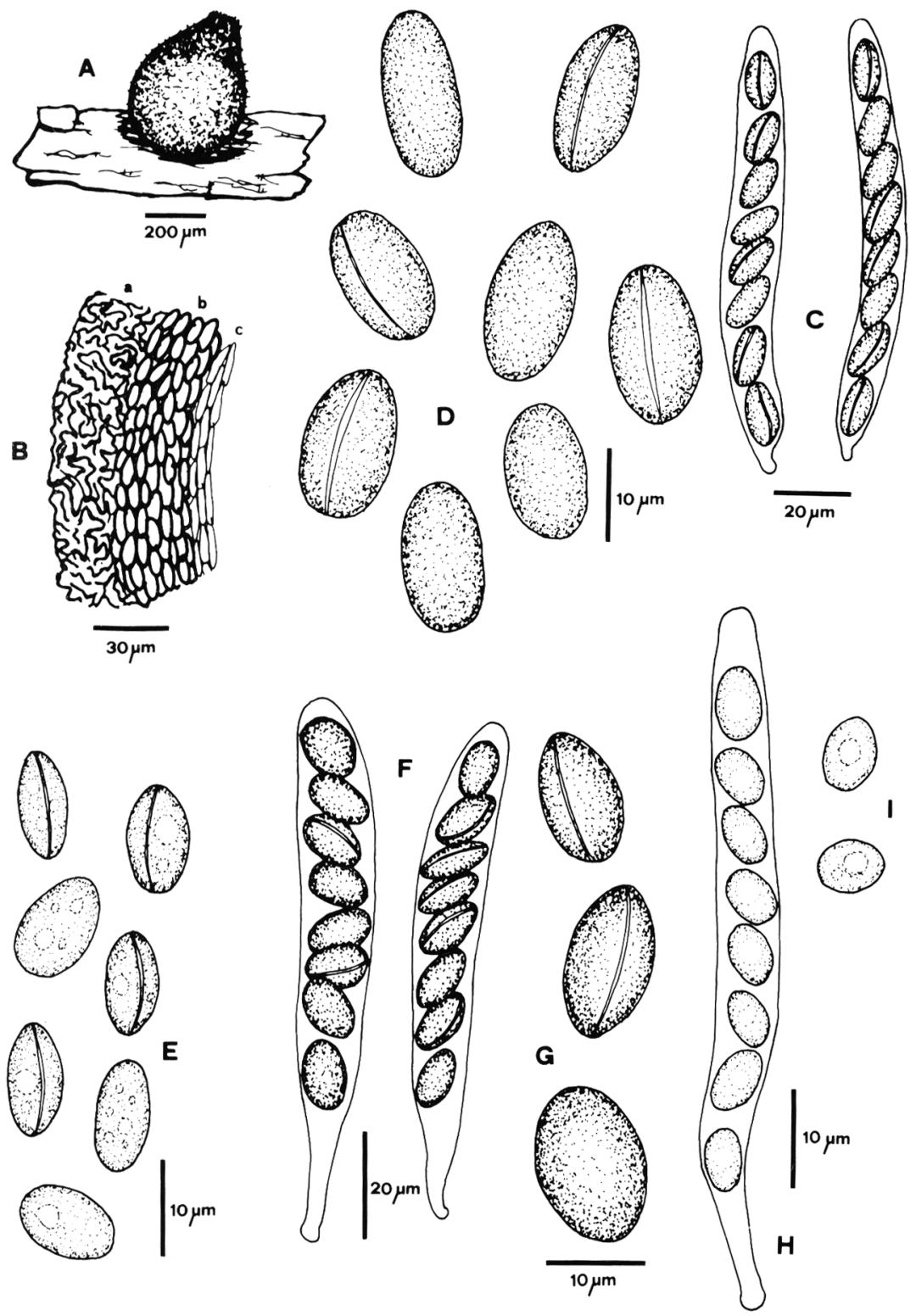

FIGURA 5. A-D: Coniochaeta sanguinolenta. A: Peritecio. B: Detalle de un corte transversal de la pared peritecial; a: capa externa, plecténquima de textura epidermoidea; b: capa central, pseudoparénquima de textura prismática; c: capa interna, pseudoparénquima. E: Coniochaeta subcorticalis: Ascósporas. F-G: Coniochaeta lignaria. F: Asco. G: Ascósporas. H-I: Coniochaeta velutina. H: Asco. I: Ascósporas. 
Helvella queletii Bres.

Material estudiado: Pinsapar de la Sierra de las Nieves, Yunquera, (Málaga). Leg. A. Aparicio \& JL. 3-V-86. JL. 7299.

Hábitat: Sobre tierra, al borde de un camino.

Observaciones: El ejemplar estudiado coincide con la descripción que Dissing (1966) detalla para este taxon.

Morchellaceae Reichemb.

Morchella elata (Fries) Boud.

Material estudiado: Pinsapar de la Sierra de Zafalgar, Grazalema (Cádiz). Leg. JL. 2-V-86. JL. 7300.

Hábitat: Sobre tierra.

Observaciones: Característica de bosques de coníferas (Dennis, 1978). Aguilera (1985) cita este taxon de Grazalema (Cádiz).

Pezizaceae Dumort.

Plicaria leiocarpa (Currey) Boud.

Material estudiado: Pinsapar de Los Reales, Sierra Bermeja, Estepona, (Málaga). Leg. JL. 1-V-86. JL. 7301.

Hábitat: Sobre tierra.

Observaciones: Los géneros Plicaria Fuck. y Peziza St. Amans, se distinguen por la forma de sus ascósporas, globosas en Plicaria y elipsoidales en Peziza (Dennis, 1978). P. leiocarpa posee ascósporas de 5-8 $\mu \mathrm{m}$ de diámetro.

\section{Pyronemataceae Corda}

Caloscypha fulgens (Pers.) Boudier (Fig. 4:A-D)

Material estudiado: Pinsapar de la Sierra de las Nieves, Yunquera (Málaga). Leg. R.M. Ros \& JL. 3-V-86. JL. 7302.

Hábitat: Sobre tierra, entre musgos.

Observaciones: Especie de primavera, sobre tierra y bajo coniferas (Dennis, 1978). Breitenbach \& Franzlin (1981) afirman que se encuentra siempre próximo a Abies. Este material se ha recolectado en un bosque de A. pinsapo.

\section{SORDARIALES}

Coniochaetaceae Chen. ex Nannf.

Coniochaeta sanguinolenta (Wallr.) Checa, Barrasa \& Moreno (Fig. $5: A-D)$

Material estudiado: Pinsapar de la Sierra de las Nieves, Ronda (Málaga). Leg. JL. 1-V-86. JL. 7226.

Hábitat: Sobre ramas muertas, descortezadas, de A. pinsapo.

Observaciones: Peritecios de 300-500 x 400-500 $\mu \mathrm{m}$, gregarios, aunque pueden aparecer solitarios, superficiales, globosos a piriformes, a simple vista de color negro y, si se miran a la lupa, se aprecia en ellos un tinte rojizo en la pared y la papila negra; cubiertos con pelos de 25-50 x 3-6 $\mu \mathrm{m}$, de color pardo oscuro, más abundantes y largos en la papila ostiolar.

Pared peritecial de 40-70 $\mu \mathrm{m}$ de grosor, formada por tres zonas bien delimitadas: una externa de 25-30 $\mu \mathrm{m}$, de color fucsia, compuesta por plecténquima de textura epidermoidea; otra zona central de 25-30 $\mu \mathrm{m}$, de color pardo oscuro formada por pseudoparénquima de textura prismática, con células de pared gruesa; y una interna de 10-15 $\mathrm{m}$, con células alargadas, hialinas.

Hamatecio formado por paráfisis de 3-4 $\mu \mathrm{m}$ de diámetro, septadas, hialinas. 


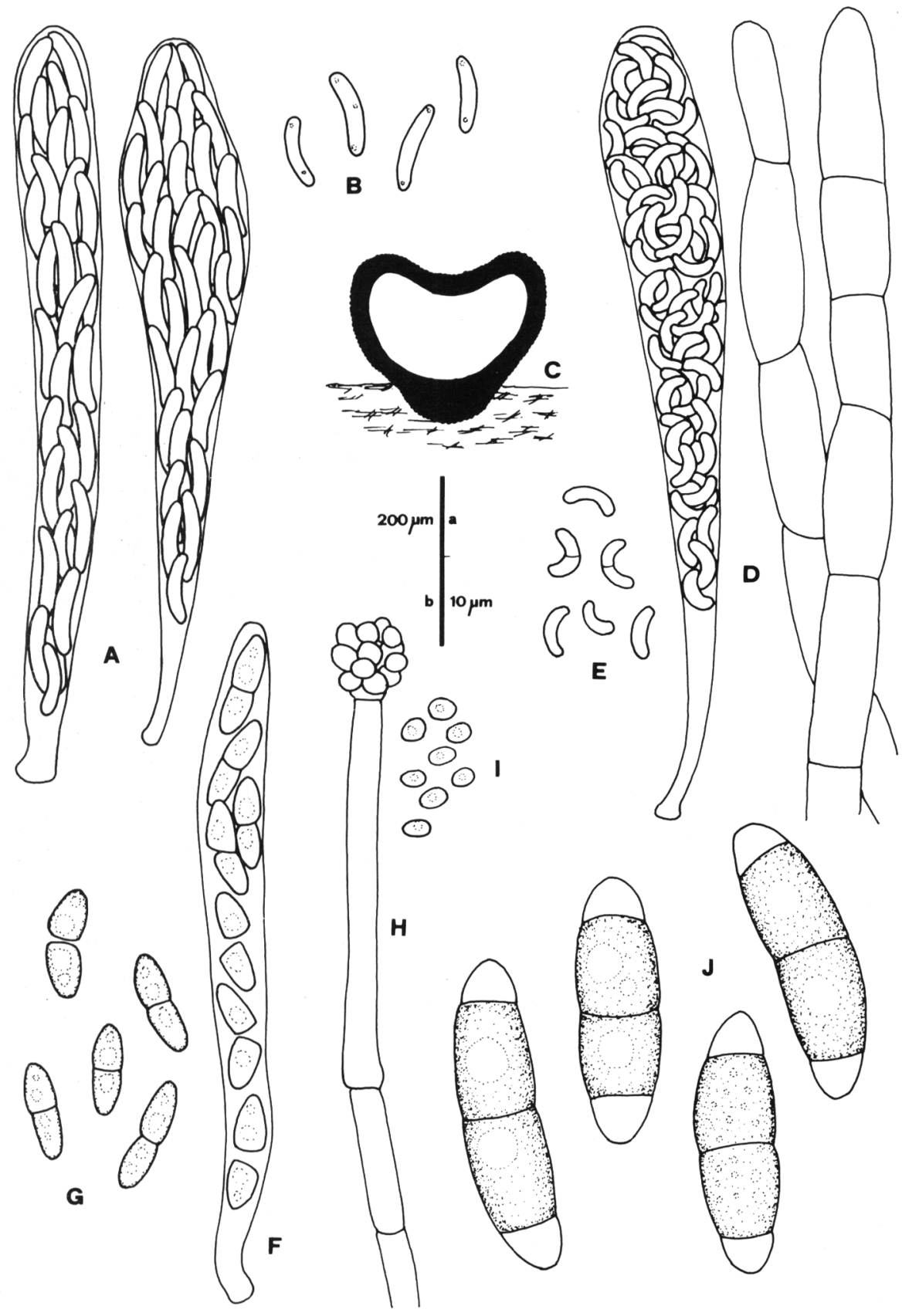

FIgURA 6. A-B: Coronophora abietina. A: Ascos. B: Ascósporas. C-E: Coronophora gregaria. C: Corte transversal de un ascoma. D: Asco y paráfisis. E: Ascósporas. F-G: Chaetosphaeria vermicularoides. F: Asco. G: Ascósporas. H-I: Chloridium sp. H: Conidióforo con conidios. I: Conidios. J: Chaetosphaerella phaeostroma: Ascósporas. Todos a escala "b" excepto C, a escala "a". 
Ascos de 90-120 x 10-12 um, cilíndricos, unitunicados, con aparato apical birrefringente, octosporados.

Ascósporas de 14-18 × 9-10 x 6-8 $\mu \mathrm{m}$, uniseriadas, elipsoidales, unicelulares, de color pardo oscuro, con fisura longitudinal.

Coniochaeta lignaria (Grev.) Massee (Fig. 5:F-G)

Material estudiado: Pinsapar de la Sierra de las Nieves, Ronda (Málaga). Leg. JL. 1-V-86. JL. 7230.

Hábitat: Sobre rama muerta, descortezada, de pinsapo.

Observaciones: Especie citada de distintos puntos de España: Se desarrolla tanto sobre madera muerta como sobre excrementos.

Coniochaeta subcorticalis (Fuck.) Munk (Fig. 5:E)

Material estudiado: Pinsapar de la Sierra de las Nieves, Yunquera, (Málaga). Leg. JL. 3-V-86. JL. 7255.

Hábitat: Sobre madera muerta, descortezada, de pinsapo.

Observaciones: El ejemplar estudiado concuerda con la descripción de Munk (1957) para C. subcorticalis. Como indican Munk (1957) y Mahoney \& Lafavre (1981), las ascósporas, de 10-12 × 6-7 × 5-6 $\mu \mathrm{m}$, son frecuentemente irregulares en forma, y a veces, se estrechan en un extremo.

Coniochaeta velutina (Fuck.) Munk (Fig. 5:H-I)

Material estudiado: Pinsapar de Los Reales, Sierra Bermeja, Estepona, (Málaga). Leg. JL. 1-V-86. JL. 7249/ Pinsapar del Cortijo de la Nava de San Luis, Ronda (Málaga). Leg. JL. 1-V-86. JL. 7250.

Hábitat: Sobre ramas muertas de A. pinsapo y Ulex baeticus.

Observaciones: Taxon caracterizado por el pequeño tamaño de sus ascósporas $(6-7 \times 4-6 \times 3-5 \mu \mathrm{m})$.

Nitschkiaceae (Fitzp.) Nannf.

Bertia moriformis (Tode: Fr.) De Not.

Material estudiado: Pinsapar de Los Reales, Sierra Bermeja, Estepona, (Málaga) 13-VI-84. JL. 7207. Pinsapar del Cortijo de la Nava de San Luis, Ronda, (Málaga) 22-XI-84. JL. 2293/1-V-86. JL. 7223. Pinsapar de la Sierra de las Nieves, Yunquera, (Málaga). 3-V-86. JL. 7254. JL. 7257/23-XI-86, JL. 7284 .

Hábitat: Sobre ramas muertas, descortezadas, de pinsapo.

Observaciones: Ascomas de 0,6-0,7 x 0,7-0,8 $\mu \mathrm{m}$, verrucosos, reconocibles a simple vista porque semejan un fruto de zarzamora.

Frecuentemente encontrada en toda la Península Ibérica, sobre madera descortezada, generalmente de Fagus sp.

Coronophora abietina Fuck. (Fig. 6:A-B)

Material estudiado: Pinsapar de Los Reales, Sierra Bermeja, (Málaga). Leg. JL. 1-V-86. JL. 7235. JL. 7238.

Hábitat: En ramas muertas, corticadas, de Abies pinsapo.

Observaciones: Peritecios de 0,4-0,7 mm de diámetro, en grupos de 3-10 situados en las cicatrices dejadas por las acículas, de color negro, colapsados lateralmente cuando secos, globosos en estado húmedo. Peridio areolado, de 110-140 $\mu \mathrm{m}$ de anchura. formado por tejido pseudoparenquimático de textura angular, con células de 10-25 $\mu \mathrm{m}$ de diámetro, de pared muy gruesa en la zona externa, que se alargan y llegan a ser hialinas en la interna.

Hamatecio ausente.

Ascos de 65-100 x 12-15 $\mu \mathrm{m}$, claviformes, en ocasiones poseen un estrechamiento en la zona apical, estipitados, unitunicados, polisporados.

Ascósporas de 8-11 x 1-2 $\mu \mathrm{m}$, alantoides, gutuladas, ligeramente curvadas, en ocasiones con un pseudosepto.

Saccardo (1882) y Berlese (1968) citan C. abietina sobre Abies 


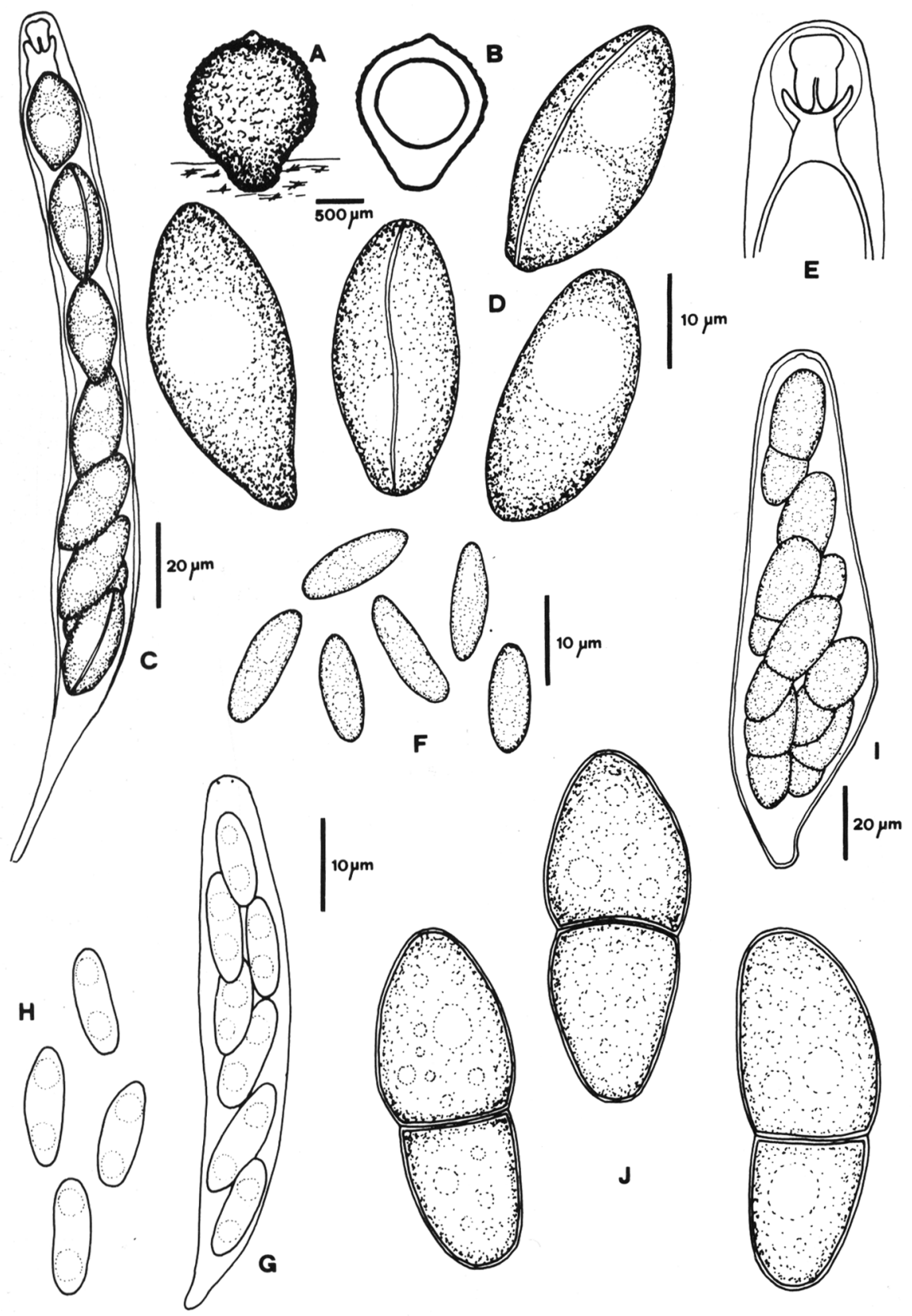

FIGURA 7. A-E: Rosellinia tassiana. A: Ascoma. B: Corte transversal de un ascoma. C: Asco. D: Ascósporas. E: Aparato apical del asco. F: Anthostoma dryophilum: Ascósporas. G-H: Endoxyla rostrata. G: Asco. H: Ascósporas. I-J: Microthelia incrustans. I: Asco. J: Ascósporas. 
excelsior y señalan que se trata de un taxon muy próximo a C. gregaria (Lib.) Fuck. Distinguen ambos taxones por la presencia de "asci abrupta clavatis, coronatis, inferiori parte multo latior: quam in C. gregaria"

Coronophora gregaria (Lib.) Fuck. (Fig. 6:C-E)

Material estudiado: Pinsapar de la Sierra de las Nieves, Yunquera, (Málaga). Leg. JL. 23-XI-84. JL. 7087.

Hábitat: Superficie interna de corteza de pinsapo.

Observaciones: Ascomas de 0,5-0,8 mm de diámetro, de dispersos a gregarios, de color negro, dispuestos en las fisuras de la cara interna de la corteza, subglobosos cuando húmedos, más altos que anchos, colapsados lateralmente o en forma de copa cuando secos, de superficie muy verrucosa. Pared formada por pseudoparénquima compuesto por células globosas en la parte externa, de 10-20 $\mu \mathrm{m}$ de diámetro, con pared muy gruesa de color pardo oscuro hacia el interior, la pared de la células se adelgaza progresivamente; las células de la capa interna son de 15-25 x 2,5-5 $\mu \mathrm{m}$, alargadas, hialinas.

Hamatecio formado por paráfisis de 5-8 $\mu \mathrm{m}$ de diámetro, de mayor longitud que los ascos, poco numerosas, hialinas, septadas, constrictas en los septos. Munk (1957) señala que esta especie no posee paráfisis, En el ejemplar estudiado, se observan paráfisis que concuerdan con la descripción que hace de ellas Saccardo (1882).

Ascos de 60-90 x 13-18 $\mu \mathrm{m}$, claviformes, de ápice obtuso, pedicelados unitunicados, polisporados. Ascósporas de 7-9 x 1,5 $\mu \mathrm{m}$, alantoides, muy corvadas, generalmente con un pseudosepto en posición central.

C. gregaria se desarrolla aislada o en grupos (Saccardo, 1882; Munk, 1957; Dennis, 1978).

Muchas de las ascósporas observadas presentan un pseudosepto; esto concuerda con lo mencionado por Munk (1957). Sin embargo, Dennis (1978) anota que las ascósporas no son septadas.

Según lo observado en el material estudiado C. gregaria se diferencia de C. abietina por: C. gregaria posee ascósporas ligeramente inferiores en tamaño y más curvadas, y sus ascos carecen de la "corona" que menciona Saccardo para C. abietina. Es posible que no exista separación a nivel específico, pero debido a las diferencias ecológicas (C. gregaria dispuesta en las fisuras del interior de la corteza del tronco y C. abietina en las cicatrices de las acículas de ramas) y morfológicas observadas, mantenemos los dos taxones citados por Saccardo (1882).

\section{TRICHOSPHAERIALES}

Trichosphaeriaceae Winter

Chaetosphaerela phaeostroma (Dur. \& Mont.) Muiller \& Booth (Fig. 6:J)

Material estudiado: Pinsapar de la Sierra de Zafalgar, Grazalema (Cádiz) Leg. 2-V-86. JL. 7214. JL. 7221.

Hábitat: Sobre rama muerta de Ulex baeticus.

Observaciones: Taxon caracterizado por sus ascósporas de $28-40$ x 8-9 $\mu \mathrm{m}$, triseptadas, con las células centrales de color pardo y las apicales, hialinas y de menor tamaño.

Chaetosphaeria vermicularoides (Sacc. \& Roum.) W. Grams \& Hol.-Jech (Fig. 6:F-I)

Material estudiado: Pinsapar del Cortijo de la Nava de San Luis, Ronda, (Málaga). Leg. JL. 1-V-86. JL. 7290.

Hábitat: Sobre ramas muertas de Ulex baeticus.

Observaciones: Ascósporas de 9-12 x 3-3,5 $\mu \mathrm{m}$, elipsoideo-fusiformes, hialinas, con un septo transversal por el que se fragmentan, incluso aún dentro del asco; una vez maduras, presentan pared gruesa, ligeramente verrucosa. 
Entre los peritecios gregarios aparecen conidióforos simples, de 20-60 $\mu \mathrm{m}$ de longitud, erectos, de color pardo, que producen conidios hialinos, de 2-3,5 x 2-2,5 $\mathrm{mm}$, los cuales se disponen en una matriz gelatinosa mucilaginosa en el extremo del conidióforo. El estado conidial corresponde al género Chloridium (Ellis \& Ellis, 1985).

\section{XYLARIALES}

Xylariaceae Tul. \& C. Tul.

Hypoxylon serpens (Pers.) Fries

Material estudiado: Pinsapar de la Sierra del Pinar, Grazalema (Cádiz). Leg. JL. 14-XI-83. JL. 7082. JL. 7759.

Hábitat: Sobre madera muerta de Quercus faginea.

Observaciones: El material estudiado responde a la descripción dada por Munk (1957) para H. serpens.

Rosellinia aquila (Fries) De Not

Material estudiado: Pinsapar de la Sierra del Pinar, Grazalema (Cádiz). Leg. JL. 2-V-86. JL. 7209.

Hábitat: Sobre ramas muertas de Quercus faginea.

Observaciones: Especie común, ampliamente distribuida en la Península Ibérica, sobre distintos sustratos.

Rosellinia tassiana De Not (Fig. 7:A-E)

Material estudiado: Pinsapar de la Sierra del Pinar, Grazalema, (Cádiz). Leg. JL. \& MH. 14-XI-83. JL. 7253.

Hábitat: Sobre corteza de un tocón de pinsapo, en la cara interna.

Observaciones: Peritecios de 1,5-2 x 2-2,5 mm de color negro, de dispersos a gregarios, superficiales, globosos; presentan pié basal, blanco internamente, rodeado de una envoltura estromática muy gruesa; de superficie verrucosa, formada en su zona exterior por tejido pseudoparenquimático $y$ en el interior por plectenquimático. Papila diminuta.

Hamatecio compuesto por paráfisis de 1,5-5 $\mu \mathrm{m}$, hialinas, tabicadas, gutuladas.

Ascos de 175-200 x 20-30 $\mathrm{m}$, cilíndricos, pedicelados, unitucados, con aparato apical I+ formado por dos anillos, octosporados.

Ascósporas de 28-34 x 13-15 $\mu \mathrm{m}$ de color pardo oscuro, uniseriados, elipsoideofusiformes a citriformes, con ranura longitudinal de germinación y 1-2 grandes gútulas.

Taxon característico por la superficie verrucosa del peritecio, con su gran tamaño y por la base columnar que sostiene al peritecio, que semeja un pié (Traverso, 1907).

Géneros de ubicación taxonómica incierta

Anthostoma dryophilum (Currey) Sacc. (Fig. 7:F)

Material estudiado: Pinsapar de la Sierra del Pinar, Grazalema (Cádiz). Leg. JL. 14-XI-83. JL. 7260.

Hábitat: En ramas muertas de Quercus faginea.

Observaciones: Peritecios en estromas pardo-verdosos en su interior, similares a los de Valsa. Ascósporas de 10-15 x 3,5 $\mu \mathrm{m}$, de color pardo, cilíndrico-elípticos, gutulados.

Generalmente sobre ramas muertas de Quercus (Saccardo, 1882; Ellis \& Ellis, 1985).

Didymella hellebori (Chaill.:Fr.) Sacc.

Material estudiado: Pinsapar de la sierra de las Nieves, Ronda, 
(Málaga). Leg. JL. 1-V-86. JL. 7265.

Hábitat: En tallos y pecíolos muertos de Helleborus foetidus. (Cádiz).

Observaciones: Citada por Bertault (1974) del pinar de San Cristóbal

Endoxyla rostrata (Tode) Munk (Fig. 7:G-H)

Material estudiado: Pinsapar de la Sierra del Pinar, Grazalema,

(Cádiz). Leg. JL. 14-XI-83. JL. 7252. JL. 7246.

Hábitat: en tocón de Abies pinsapo.

Observaciones: El material estudiado concuerda con la descripción dada por Munk (1957), para esta especie.

Microthelia incrustans (Ell. \& Ev.) Corlett \& Hughes (Fig. 7:I-J).

Material estudiado: Pinsapar de Los Reales, Sierra Bermeja, Estepona, (Málaga). Leg. MH. 14-III-84. JL. 7064. Pinsapar de la Sierra del Pinar. Grazalema, (Cádiz). Leg. JL. 2-V-86. JL. 7220.

Hábitat: Sobre madera muerta de pinsapo.

Observaciones: Según Hawksworth et al. (1983), el género Microthelia Körber debe considerarse como sinónimo de Anisomeridium Choisy. Eriksson \& Hawksworth (1985) no reconocen el género Microthelia, e incluyen Anisomeridium en la familia Strigulaceae Fries, a la cual estiman de posición taxonómica incierta. Otros autores (Sivanesan, 1984; Ellis \& Ellis, 1985) mantienen el nombre genérico propuesto por Körber. En este sentido, mantenemos en el presente trabajo el binomio Microthelia incrustans, debido a que es el único observado en la bibliografía consultada, para la denominación de este taxon.

En el material estudiado, las ascósporas son de tamaño superior al indicado por Sivanesan (1984): 35-40 x 14-15 $\mu \mathrm{m}$ y 22-27-(37) x 7-8-(12,5) $\mu \mathrm{m}$ respectivamente. Sin embargo, Ellis \& Ellis (1985) señalan dimensiones entre 25-35 x 8-11 $\mu \mathrm{m}$, y se refieren a que las ascósporas de este taxon son muy variables en tamaño, pudiendo alcanzar hasta 45 × $15 \mu \mathrm{m}$.

\section{BIBL IOGR AF I A}

AGUILERA, A. -1985- Estudios sobre el orden Pezizales (AScomycotina) en Andalucía. Tesis de Licenciatura Inéd. Universidad de Granada.

ARX, J.A. VON \& MULLER, E. -1954- Die Gattungen der Amerosporen Pyrenomyceten. Beitr. Kryptogamenfl. Schwiz. 434 p.

ARX, J.A. VON \& MULLER, E. -1975- A re-valuation of the bitunicate Ascomycetes with keys to families and genera. Stud. Mycol. 9:1-159.

BARR, M.E. -1972- Preliminary studies on the Dothideales in Temperate North America. Contribution from the University of Michigan Herbarium $9(8): 523-638$.

BARR, M.E. -1982- On the Pleomassariaceae (Pleosporales) in North America. Mycotaxon 15:349-383.

BERLESE, A.N. -1894/1905- Icones Fungorum. Vol. I-III. (Reprint 1968). J. Cramer, New York.

BERTAULT, R. -1974- Contribution à la Flore Mycologique de l'Andalousie. Collectanea Botánica 9(2):25-44.

BooTH, C. -1959- Studies of Pyrenomicetes, IV. Nectria (part. I)Mycological Papers, 73-115 p.

BREITENBACH, J. \& FRANZLIN, F. -1981- Champignons de Suisse. Tome 1: Les Ascomycètes. Ed. J. Breitenbach et F. Fränzlin, Societé de Mycologie de Lucerne. $310 \mathrm{p}$. + $390 \mathrm{pl}$.

CHESTERS, C.G.C. \& BELL, A. -1970- Studies in the Lopuiostomataceae Sacc. Mycological Papers, $120 \mathrm{p}$.

DENNIS, R.W.G. -1978- British Ascomycetes. J. Cramer, Vaduz. 585 p. 
DISSING, H. -1966- A revision of collections of the genus Helvella L. ex St. Amans emend. Nannf. in the Boudier Herbarium. Revue de Mycol. $31(3): 189-228$.

ELLIS, M.B. \& ELLIS, J.P. -1985- Microfungi on Land Plants. Ed. Croom Helm. London $818 \mathrm{p}$.

ERIKSSON, O. \& HAWKSWORTH, D.L. -1985- Outline of the Ascomycetes. Systema Ascomycetum, 4:1-79.

HAWKSWORTH, D.L., SUTTON, B.C. \& AINSWORTH, G.C. -1983-Dictionary of the Fungi. Surrey, $445 \mathrm{p}$.

HOLM, L. HOLM, K. -1977- Nordic junipericolous Ascomycetes. Acta Univ. Uppsal. Symb. Bot. Uppsal. XXI:3. Uppsala. 70 p.

LARIOS, J.M., HONRUBIA, M. \& MORENO, G. -1986- Estudio de los hongos que fructifican en la vegetación relicta de Abies pinsapo Boiss., en España Peninsular. I. Ascomycotina. Acta Bot. Malac. 11:39-54.

MAHONEY, D.F. \& LAFAVRE, J.S. -1981- Coniochaeta extramundana, with a synopsis of other Coniochaeta species. Mycologia, 73(5):931-952.

MIRZA, F. -1968- Taxonomic investigations on the Ascomycetous Genus Cucurbitaria S.F. Gray. Nova Hedwigia, 16:161-213.

MUNK, A. -1957+ Danish Pyrenomycetes. Dansk. Bot. Arkiv. 17:1-491.

SACCARDO, P.A. -1882/1883- Sylloge Fungorum. Vols. I-II. (Reprint 1966). Johnson Reprint Corporation. New York.

SIVANESAN, A. -1984- The Bitunicate Ascomycetes and their anamorphs. J. Cramer. $701 \mathrm{p}$.

TRAVERSO, J.B. -1907-Flora Italica Cryptogama. Vol. II. Ed. Capelli, 353-492.

WEHMEYER, L.E. -1975- The Pyrenomycetous Fungi. Mycol Mem. 6:1-250.

ZOGG, H. -1962- Die Histeriaceae und Lophiaceae. Beitr. Kryptogammenfl. Schweiz. 11(3):1-190.

(Aceptado para su publicación el 27 de septiembre de 1987)

Dirección de los autores: J.M. LARIOS \& M. HONRUBIA: Departamento de Botánica, Universidad de Murcia. G. MoReno: Departamento. de Botánica, Universidad de Alcalá de Henares. 
